\title{
Investigación
}

\section{Las acciones colectivas del movimiento feminista de Bogotá frente al caso de Rosa Elvira Cely 2012-2015}

\section{Collective actions of the feminist movement held in Bogotá in the case of Rosa Elvira Cely 2012-2015}

Lina Marcela Ayala Morales ${ }^{2}$

Recepción: 20/01/2020 • Aprobación: 06/04/2021 • Publicación: 17/11/21

Para citar este artículo

Ayala Morales, L. M. (2021). Las acciones colectivas del movimiento femi-

nista de Bogotá frente al caso de Rosa Elvira Cely 2012-2015. Dos mil tres

mil, 23, e216. https://doi.org/10.35707/dostresmil/23216

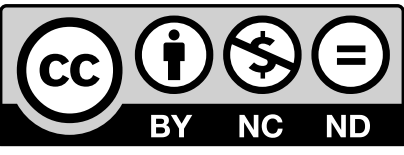

${ }^{1}$ El presente artículo es derivado de un trabajo de grado ya finalizado del programa de Ciencia Política de la Universidad de Ibagué: Las acciones colectivas del movimiento feminista de Bogotá frente al caso de Rosa Elvira Cely 2012-2015 (Ayala, 2019).

${ }^{2}$ Programa de Ciencia Política, Universidad de Ibagué, Ibagué, Colombia. Correo electrónico: marcelaayalam@gmail.com 
Resumen. El presente artículo reconoce la importancia de los movimientos sociales y cómo estos generan acciones ante la violencia de género, en específico, los feminicidios que padecieron las mujeres en Bogotá. Un caso que ha sido relevante dentro de la historia de Colombia, por el grado de agresión de la víctima, es lo sucedido con Rosa Elvira Cely en Bogotá en el 2012. De esta manera, se pretende resaltar el papel que jugó el movimiento feminista bogotano y las acciones colectivas presentadas para exigir justicia y protección a las víctimas, sobrevivientes y sus familias.

La metodología empleada fue un estudio de caso al considerar la adecuada interpretación y la relación de los grupos, colectivos, fondos y demás involucrados en el movimiento, en tanto a la manera de proceder y los logros obtenidos como resultados de las acciones colectivas presentadas. Es necesario aclarar que la parte jurídica no será tema de estudio, pero sí es parte del resultado del accionar del movimiento feminista bogotano.

Abstract. This article recognizes the importance of social movements and how they generate actions against gender violence, specifically, the femicides suffered by women in Bogotá. A case that has been relevant in the history of Colombia, due to the degree of aggression of the victim, was what happened to Rosa Elvira Cely in Bogotá in 2012. In this way, it is intended to highlight the role played by the feminist movement in Bogotá and the collective actions presented to demand justice and protection for victims, survivors, and their families.

The methodology used was a case study considering the proper interpretation and relationship of the groups, collectives, funds and others involved in the movement, in terms of the way of proceeding and the achievements obtained as results of the collective actions presented. It is necessary to clarify that the legal part will not be the subject of study, but it is part of the result of the actions of the feminist movement in Bogotá.

\section{Palabras claves}

Movimiento feminista, acciones colectivas, feminicidio.

Key words

Feminist movement, collective actions, femicide. 


\section{Introducción}

Es menester investigar sobre el feminicidio de Rosa Elvira Cely, ya que es una situación que han sufrido muchas mujeres en Bogotá y en el resto de Colombia. Se cree que al denunciar por años esta clase de crímenes no se presta la atención necesaria, reflejando la negligencia del Estado. Además, la muerte de Rosa Elvira marcó el desarrollo de la concientización de los colombianos indignados ante todo tipo de violencia contra la mujer en nuestro país.

Asimismo, es importante analizar la participación de las mujeres y las acciones desatadas por el caso de Cely para destacar los esfuerzos multisectoriales de cada uno de los grupos, colectivos, fondos y demás personas en general que se unieron al movimiento de mujeres o feministas de Bogotá. Todos estos involucrándose en procesos para lograr justicia y la manera en que la sociedad colombiana generó respuestas frente a las diversas acciones colectivas manifestadas en este caso durante el periodo 2012-2015 en Bogotá, Colombia.

$\mathrm{Al}$ mismo tiempo, resaltan los logros que sobresalieron de intervenciones para exigir justicia frente a problemáticas que pusieron en riesgo la vida de la mujer y, de esta manera, enfatizar cómo el proceso avanzó hasta llegar a la tipificación de las muertes a mujeres por la condición de serlo, como feminicidio en la legislación colombiana, sin entrar en campos jurídicos.

De esta forma, se busca destacar cómo la sororidad de estos grupos de mujeres y cómo sus acciones colectivas lograron tener un cambio en el paradigma no solo en Bogotá, sino en toda Colombia, ante una sociedad considerada machista, la cual era y, aun después de tipificaciones en pro de los derechos de las mujeres, sigue siendo impune ante los diversos ataques a mujeres en distintas circunstancias. Así, animar no solo a mujeres sino a la sociedad entera a exigir y luchar por la justicia y equidad, para que puedan vivir en condiciones dignas y romper las barreras de violencia de género establecidas en Colombia.

Las consideraciones expuestas me llevan a generar las siguientes preguntas orientadoras de la investigación: ¿Cuáles fueron los grupos, colectivos o fondos que se unieron al movimiento feminista bogotano y que intervinieron ante la muerte de Rosa Elvira Cely?, ¿cuáles fueron las acciones colectivas presentadas por el movimiento feminista bogotano? y ¿qué logros y beneficios trajo este accionar para las colombianas? De esta manera, los objetivos son reconocer y analizar las acciones colectivas del movimiento feminista bogotano que participó a raíz de la muerte de Rosa Elvira Cely en Bogotá, Colombia, durante el 2012 -2015. 


\section{Rosa Elvira Cely}

He intentado imaginarme varias veces de qué moriré. ¿Sufriré un ataque al corazón? ¿Padeceré una enfermedad atroz? ¿Me asesinará un hombre? Natsuo Kirino (2003)

En Colombia podemos mencionar el poco interés del Estado frente a crímenes de extrema violencia contra las mujeres; tal fue el caso de Rosa Elvira Cely, mujer de 35 años, que sufrió un atroz suceso en el cual fue violada y empalada; presentaba, además, señales de una brutal agresión con arma blanca, todo esto cometido en el Parque Nacional de Bogotá el día 23 mayo del 2012. Rosa Elvira, luego de terminar su jornada académica en horas de la noche, decidió salir con sus compañeros del Colegio Manuela Beltrán donde validaba su bachiller académico, al final accedió a que uno de ellos la llevara hasta su casa sin saber el rumbo que tomaría Javier Velasco y su trágico desenlace. Es allí donde comienza el calvario de Rosa Elvira, quien fue sometida a la voluntad de Javier Velasco y quien luego de este terrible e indignante suceso terminó por dejarle el celular en el pecho. Fue ahí, en medio del dolor y del frío, en el Parque Nacional donde Rosa Elvira tomó fuerza y llamó a la línea de emergencia 123 para solicitar ayuda debido a la gravedad de la agresión.

El personal que recibió la llamada creyó que era una llamada de broma y, luego de varios intentos por mantener la conversación, revictimizan a Rosa Elvira preguntándole que si estaba tomada ${ }^{3}$. La llamada es el inicio de la negligencia presentada en este caso, dada la duración del tiempo de respuesta y de comprensión de la situación, además del tiempo de acción de la policía, quienes tardaron dos horas en hallarla, el mismo tiempo que demoró la ambulancia en llegar al sitio de la tragedia. Aunque el tiempo transcurrido desde la llamada de ayuda es extenso, Rosa Elvira es encontrada con vida y es en medio del chequeo médico que logra expresar lo sucedido y señalar a los dos culpables de esta tragedia.

En este punto, se observó la segunda falla por parte del Estado. La atención por parte de las instituciones de salud no fue la mejor, ya que se dio la orden al personal de la ambulancia de llevarla al Hospital Santa Clara, ubicado al otro lado del distrito, teniendo cerca al Hospital San Ignacio y al Hospital Militar. Cuando llegaron al Hospital Santa Clara el proceso de atención médica a Rosa Elvira no fue el adecuado, a pesar de la gravedad, fue obligada a esperar en una camilla en un espacio alejado debido a que el pasillo estaba lleno de pacientes esperando ser atendidos. Rosa Elvira, durante la espera, llamó a la enfermera para indicarle que tiene ganas de

\footnotetext{
${ }^{3}$ De esta manera Fernando González Santos en su libro La vida es Rosa: el oscuro amanecer de Rosa Elvira en el Parque Nacional, menciona la llamada de Rosa Elvira a la línea de emergencia 123.

¡Por favor, urgente! ¡Ayúdeme! ¡Por favor! ¡Por favor!

¿Usted se encuentra tomada?

¡Por favor, ayúdeme! No me puedo mover...
} 
ir al baño y fue allí donde entró en paro, fue en este acontecimiento que le dieron importancia a su situación y le brindaron la atención médica necesaria ${ }^{4}$.

Al difundirse lo sucedido se genera gran polémica y conmoción en todo el país, es así que diversos medios de comunicación iniciaron la tarea de informar lo ocurrido, entre ellos, la revista Semana que emite una nota judicial sobre el horripilante crimen y menciona que:

Rosa Elvira sufrió un paro cardíaco, perdió la conciencia y al ser intervenida en el quirófano le encontraron la pelvis y el útero rotos como consecuencia de un palo que le habían introducido por el ano. Dentro del cuerpo se hallaron rastros de yerba y astillas. Fueron cinco días de lucha en cuidados intensivos. Los médicos probaron, infructuosamente, distintas maniobras para controlar la infección interna que sobrevino, así como el traumatismo craneoencefálico. (Semana, 2012a).

A tal punto que no logró sobrevivir, ya que el tortuoso maltrato ocasionó lesiones que disminuyeron su función vital. Por consiguiente, este comunicado explica la gravedad del asunto, además, cabe resaltar el impacto del personal hospitalario del Hospital Santa Clara al examinar el cuerpo de Rosa Elvira y la melancolía de una gran parte de Colombia ante los hechos ocasionados a Cely.

Días después del fallecimiento y la divulgación del crimen ocasionado a Rosa Elvira, el domingo 3 de junio en Bogotá, se reunieron miles de personas en horas de la mañana en el Parque Nacional, lugar en donde ocurrieron los hechos, manifestando el rechazo y repudio contra esta clase de crímenes, a los cuales son sometidas algunas mujeres en Colombia, en donde la negligencia y la inasistencia de las instituciones del Estado reflejan la despreocupación de este tipo de eventos. Entre los manifestantes del evento se encontraban sus familiares, amigos, compañeros y profesores de la jornada nocturna del Colegio Manuela Beltrán, estudiantes y egresados de la Universidad Nacional y demás personas de la sociedad civil que por medio de $\operatorname{cartas}^{5}$, carteles, panfletos, música y oraciones refutaban esta clase de actos y exigían justicia para Rosa Elvira y las demás mujeres que han sido víctimas de esta clase de crímenes que han quedado en la impunidad colombiana (Notiagen, 2012).

Por consiguiente, es menester abordar este tema de investigación ya que la muerte de Rosa Elvira Cely y el impacto que ocasionó, motivó a la sociedad para que luchara y realizara manifestaciones por todos los casos de mujeres víctimas de crímenes pasionales, en un país donde la justicia no les brindaba la adecuada atención a las mujeres frente a estos casos. Es importante resaltar que dentro de la sociedad colombiana ya existían y a la vez se crearon campañas y grupos que luchaban y exigían justicia ante estas problemáticas, que vulneran los derechos y la dignidad de la mujer. De esta forma, se busca resaltar el papel del movimiento feminista

\footnotetext{
4 "La enfermera le informa que cuando estaban llevando a Rosa Elvira al baño en una silla de ruedas, entró en paro. Por eso debieron trasladarla de emergencia a sala de reanimación. Al revisar sus órganos genitales, se dieron cuenta que se trataba de un hecho realmente temerario, ante el cual no era seguro si la paciente iba a reaccionar positivamente” (González Santos, 2014).

${ }^{5}$ Como lo fue la carta al Estado y a la sociedad colombiana y al mundo "Por el derecho a la vida ¡Ni una RosA más!”
} 
bogotano, quienes intervinieron con acciones colectivas para llevar a cabo un cambio del paradigma establecido en Colombia.

De esta manera, es importante que toda agresión hacia las mujeres requiera de una mayor intervención por parte del Estado para que no quede en la impunidad. Además, establecer este delito, tal como ocurrió después de la manifestación del movimiento feminista bogotano, al exigir justicia para Rosa Elvira y para todas las mujeres que han sido agredidas en casos que quedaban impunes.

\section{El victimario}

Frente a esta situación es importante mencionar al victimario, Javier Velasco Valenzuela, compañero de estudios de Rosa Elvira, quien fue uno de los actores materiales y desde el inicio uno de los posibles culpables de la muerte de Rosa Elvira. Velasco ha sido uno de los criminales más recordados en Colombia, no solo porque acabó con la vida de Rosa Elvira Cely, sino que marcó la historia en el país, ya que demuestra que la justicia en Colombia no actúa frente a los inicios de un agresor para las mujeres y es negligente en generar penas y castigos, dejando en la impunidad a posibles feminicidas que atentan diariamente a las colombianas.

La negligencia del Estado, en primera instancia, se reflejó en el 2003 cuando Velasco asesinó a Dismila Ochoa a machetazos, este salió ileso de la situación ya que logró que lo declararan inimputable por supuesta enfermedad psiquiátrica, la cual fue un medio de escape a este delito; la justicia colombiana, tras un tratamiento de seis meses, lo dejó en libertad. Asimismo, en 2008 violó a una trabajadora sexual ya que ella se negó a tener relaciones sexuales sin condón con Velasco, motivo por el cual le propició una inclemente golpiza, dichas situaciones dejaron a un hombre violento en libertad y disfrutando de la potestad de burlar a la justicia colombiana sin pena alguna, hasta el 2012 con lo sucedido con Rosa Elvira. A su historial se le sumó el acceso carnal violento de sus hijastras (El Tiempo, 2016).

\section{Marco teórico}

Al hablar de los movimientos sociales es necesario mencionar la teoría de los nuevos movimientos sociales en la que se encuentra el feminismo; también, establecer qué son las acciones colectivas y el feminicidio. Por consiguiente, se resalta el papel que ha generado el movimiento feminista frente a los feminicidios de los cuales sus acciones generaron resultados que trasformaron la historia de la mujer desde épocas remotas hasta la actualidad, de esta forma, la mujer asumió protagonismo y buscó la emancipación en distintos planos de la vida.

Por todos los hechos discriminatorios hacia las mujeres, estas tomaron conciencia de manera colectiva ante estas situaciones que vulneraban su dignidad y sus derechos en la sociedad, para exigir ser tratadas como personas en igualdad de condiciones. Por ello, fueron diversos los acontecimientos que motivaron y animaron a mujeres a organizarse para luchar contra un 
paradigma transmitido de generación en generación, para así cambiar el rumbo de sus vidas y para las futuras generaciones.

En este sentido, los movimientos sociales son uno de los medios más sobresalientes para denunciar injusticias y presionar para la realización de trasformaciones sociales, culturales, democráticas y jurídicas, demostrando su fuerza para generar un cambio social y, de esta manera, cambiar el rumbo de la historia de las mujeres.

De acuerdo con lo anterior, los movimientos sociales son aquellas construcciones sociales, culturales, jurídicas y políticas establecidas por un grupo de personas que en su mayoría son vulneradas en algún plano de la vida, que tienen uno o varios motivos para organizarse y exigir un cambio dentro de la sociedad, por medio de acciones colectivas que logran incidir y transformar situaciones mediante una organización estructurada, con una metodología de proceder establecida entre sus partes, para así lograr modificar o evitar un acontecimiento en la sociedad.

De acuerdo con las consideraciones mencionadas sobre los movimientos sociales, la teoría que mejor se adapta a la investigación es la de los nuevos movimientos sociales. Esta es guiada por valores posmaterialistas, en contraparte a los anteriores enfoques que resaltaban el valor material. Los nuevos movimientos surgen en los años sesenta como respuesta a todas las necesidades que exigían los nuevos contextos en la sociedad. Así, los nuevos movimientos reflejaron la discordancia y reprobación de la ciudadanía abriendo nuevos caminos dentro de su forma de accionar, en estos se ven plasmados diferentes ideales y nuevas integraciones de lucha, como lo fueron: las luchas de obreros, el ecologismo, el pacifismo, los antimilitarismos, el feminismo y otras más que se fueron formando de acuerdo a los problemas de la actualidad.

Según lo expuesto sobre los nuevos movimientos sociales, se menciona para la investigación que son aquellos movimientos basados en las nuevas necesidades que surgen en la población, es decir, de los fenómenos que se presentan de acuerdo con las problemáticas que afectan el proceso de la consolidación de sociedades guiadas a la modernidad, todo esto basado en valores posmaterialistas, en donde el valor del capital pasa a un segundo plano, dándole prioridad a temáticas que afectan a gran parte a la población. Todo esto sustentado en la consolidación de los derechos humanos, incentivando la democracia, la solidaridad y brindando la interacción de participar y dialogar políticamente con el gobierno frente a diversas situaciones que vulneran y desestabilizan a gran parte de la ciudadanía.

Entre la teoría de los nuevos movimientos se encuentra el movimiento feminista, cuyo objetivo en las sociedades modernas fue la lucha de igualdad de los derechos de hombres y mujeres. Al hablar del feminismo se establecen unas etapas de este movimiento social, de esta manera, en la antigüedad las mujeres fueron conscientes de la situación de inferioridad presentada en las sociedades donde el hombre contó con privilegios que sobresalían ante la situación de la mujer. Las mujeres en su intento de generar un cambio realizaban comentarios o manifestaciones por estas inconformidades, pero no fueron suficientes ni se les prestó atención necesaria a sus desconciertos. 
De esta manera, el feminismo se estableció como una corriente del pensamiento en pro de los derechos de las mujeres frente a las situaciones de discriminación presentadas en el tiempo, resaltando el papel de la mujer como un ser en iguales condiciones que los hombres. Según Varela (2008), en su libro Feminismo para principiantes, establece que el feminismo es un discurso político que se basa en la justicia para las mujeres que han sido vulneradas a través de los años; mejor dicho, que el feminismo:

Es una teoría y práctica política articulada por mujeres que tras analizar la realidad en la que viven toman conciencia de las discriminaciones que sufren por la única razón de ser mujeres y deciden organizarse para acabar con ellas, para cambiar la sociedad. Partiendo de esa realidad, el feminismo se articula como filosofía política y, al mismo tiempo, como movimiento social. (Varela, 2008, p. 10).

Entre muchos más conceptos que resaltan la toma de conciencia de la sociedad ante la situación del ser mujer para exigir respeto y resaltar su rol como un ser en iguales condiciones y que debería contar con los mismos privilegios del hombre.

\section{Acciones colectivas}

La acción colectiva es un acto fundamental en la conformación de los movimientos sociales. En el momento en que se establece un número de personas con un fin determinado forman acciones de manera colectiva, pero estas requieren una gran variedad de actitudes e intereses en común hacia una misma lucha que especifique su unión y motivación. De esta manera, las acciones colectivas son:

Acciones realizadas por un conjunto de sujetos motivados por unos intereses comunes, que adoptan una forma de organización más o menos estructurada, y diseñan unas prácticas de movilización concretas, actuando en una estructura de oportunidad política que facilitará o dificultará la acción y condicionará sus posibilidades de influir en la articulación del poder. (Funes \& Monferrer, 2003, p. 23).

Por ende, se debe resaltar la capacidad de las acciones colectivas ya que:

La acción colectiva se emplea para comunicar y trasmitir las exigencias de los Movimientos Sociales, pues supone una exteriorización de demandas que de otro modo quedarían silenciadas; generar solidaridad e identidad entre los miembros y para vincular los líderes con sus seguidores, convencer a los participantes que son más fuertes de lo que son y generar cierto simbolismo del que emana una determinada identidad: desafiar a sus adversarios a partir de la creación de la incertidumbre debido a lo indeterminado de su coste y al desafío que suponen las acciones que organizan. (Ibarra, Martí, \& Gomá, 2002, p. 36).

Así, las acciones colectivas realizadas por el movimiento feminista en Colombia lograron el inicio de unas transformaciones en una sociedad donde los asesinatos a mujeres eran silenciados y, en la cual, a partir de mayo de 2012, se inició una concientización por las situaciones a las que las mujeres estaban expuestas. 
Feminizando el mundo, a través de interacciones colectivas, ya sea a través de manifestaciones públicas o mediante acciones organizativas, en los cuales los feminismos de todos los tipos, con intermitente continuidad, han denunciado las injusticias, las deficiencias políticas y las contradicciones de la democracia. (Perrot, 1997, citado por Sandoval Acosta, 2012, p. 67).

El movimiento feminista interactuó en un mismo fin para lograr que sus acciones colectivas tuvieran como resultado el bienestar de las colombianas.

\section{Feminicidio}

De esta manera, se resalta la violencia de género que conlleva a la muerte que sufren las mujeres alrededor de la historia. Aunque antiguamente no se reconocía bajo el término de feminicidio sino de uxoricidio, que explicaba las muertes de mujeres a manos de sus esposos motivados por los celos y porque la esposa podía ser proclive a tener actitudes de adulterio.

Por consiguiente, surge el término femicidio para definir la situación de muerte de mujeres por motivos misóginos en donde no solo sus familiares sino también desconocidos imponen su voluntad ante las mujeres, de esta manera, Janet Caputi y Diana Russell establecen que el femicidio es "la forma más extrema de terrorismo sexista, motivada por odio, desprecio, placer o sentido de propiedad sobre una mujer" (Caputi \& Russell, 1992, p. 15).

Las autoras Jane Caputi y Diana Russell (1992) involucran muchas más variables sobre la violencia de género y establecen que este fenómeno:

Representa el extremo de un continuum de terror antifemenino que incluye una amplia variedad de abusos verbales y físicos, tales como violación, tortura, esclavitud sexual (particularmente por prostitución), abuso sexual infantil incestuoso o extra-familiar, golpizas físicas y emocionales, acoso sexual (por teléfono, en las calles, en la oficina, y en el aula), mutilación genital (clitoridectomías, escisión, infibulaciones), operaciones ginecológicas innecesarias (histerectomías), heterosexualidad forzada, esterilización forzada, maternidad forzada (por la criminalización de la contracepción y del aborto), psicocirugía, negación de comida para mujeres en algunas culturas, cirugía plástica y otras mutilaciones en nombre del embellecimiento. Siempre que estas formas de terrorismo resultan en muerte, se convierten en feminicidio. (Caputi \& Russell, 1992, p. 15).

Al llegar el término femicidio a América Latina se traduce de dos maneras: femicidio y feminicidio que en común se define como la muerte violenta de mujeres, pero que se diferencia por la responsabilidad estatal. Este es un tema que logra discusiones académicas sobre la utilización del término en los países, una de las grandes líderes feministas sobre el femicidio es Marcela Lagarde, quien en 1994 en México señala que el femicidio sería traducido como feminicidio, ya que el femicidio solo incluye un asesinato a las mujeres y deja sin tocar las variables que este afecta y establece que será denominado feminicidio ya que "se trata de una fractura del Estado de derecho que favorece la impunidad. El feminicidio es un crimen de Estado" (Lagarde, 2009, p. 155). Por consiguiente, el Estado tiene gran responsabilidad de que sucedan este tipo 
de eventos, ya que no brinda la seguridad y protección necesaria para que las mujeres puedan tener una vida digna y libre de violencias (Iribarne, 2015).

De la misma forma, Julia Monárrez menciona que:

El feminicidio comprende toda una progresión de actos violentos que van desde el maltrato emocional, psicológico, golpes, los insultos, la tortura, la violación, la prostitución, el acoso sexual, el abuso infantil, el infanticidio de niñas, las mutilaciones genitales, la violencia doméstica, y toda política que derive en la muerte de las mujeres, tolerada por el Estado. (2002, p. 286).

Asimismo, Monárrez, frente al contexto de la situación en Ciudad Juárez y al tratar de entender y explicar el aumento de las muertes a mujeres y la impunidad presente por parte del Estado, retoma una clasificación del feminicidio:

Feminicidio familiar (íntimo e infantil), feminicidio sexual sistémico (organizado o desorganizado) y feminicidio por ocupaciones estigmatizadas (realizadas por mujeres), y lo conceptualiza como el asesinato de mujeres por razones asociadas con su género, considerándole como una forma extrema de violencia de género, que se ejerce por los hombres contra las mujeres en su deseo de obtener poder, dominación o control. (Huertas \& Jiménez, 2016, p. 112).

\section{Metodología}

Esta investigación se realizó de acuerdo al enfoque cualitativo de las ciencias sociales, por medio del cual se busca obtener un resultado de manera explícita sobre el análisis de la participación de las mujeres colaboradoras del movimiento feminista bogotano y la manera en que sus acciones colectivas dejaron un logro en nuestro país.

El presente trabajo aborda el enfoque de los estudios de caso. Yin (1989), uno de los autores más importantes sobre el estudio de caso, establece que este consiste en "una investigación empírica que investiga un fenómeno contemporáneo en su contexto real, donde los límites entre el fenómeno y el contexto no se muestran de forma precisa, y en él, que múltiples fuentes de evidencia son utilizadas" (Yin, 1989, citado en Villareal \& Landeta, 2010, p. 33).

El autor considera que los temas de los estudios de caso son relativamente nuevos y novedosos para la sociedad y establece que tienen unas características en particular (Yin, 1989); examina o indaga sobre un fenómeno contemporáneo en su entorno real, las fronteras entre el fenómeno y su contexto no son claramente evidentes, se utilizan múltiples fuentes de datos, puede estudiarse tanto un caso único como múltiples casos.

De este modo, se define que:

El caso o los casos de un estudio pueden estar constituidos por un hecho, un grupo, una relación, una institución, una organización, un proceso social, o una situación o escenario específico, construido a partir de un determinado, y siempre subjetivo y parcial, recorte empírico y conceptual de la realidad social, que conforma un tema o problema de investigación. (Neiman \& Quaranta, 2006, p. 218). 
Es decir, que se adapta el estudio de caso al presente tema de investigación, ya que se involucra con la realidad social presentada en Colombia por el caso de Rosa Elvira, que fue un hecho que incentivó a diversas organizaciones para generar un proceso social contra la violencia de género. De igual forma, aclarar que la acumulación de la indagación se lleva adelante a partir de una estrategia que se organiza como respuesta a la pregunta de investigación. Aparte de destacar que en los estudios de caso hay una gran variedad en las fuentes de información como lo son la observación, entrevistas, documentos, entre otros, orientados a lograr como resultado una descripción compleja del fenómeno o fenómenos a estudiar (Neiman \& Quaranta, 2006).

\section{Resultados}

El fundamento del trabajo se enfoca en la situación que vivió Rosa Elvira Cely y el accionar que realizó el movimiento feminista en Bogotá por la indignación que causó tal hecho, el país quedó conmovido y de luto por una muerte con tal grado de brutalidad. Es ahí donde el Movimiento decidió accionar desde aquel mayo del 2012, ya que corresponde al mes que paralizó a los medios de comunicación y a la sociedad colombiana por el crimen de Rosa Elvira.

Lo sucedido el 24 de mayo de 2012 a Rosa Elvira Cely causó un gran impacto a nivel nacional no solo por la gravedad del ataque recibido, sino por ser en la capital del país en una zona de gran importancia para la ciudad, ya que es en el centro norte de Bogotá, en donde diariamente transitan muchos de los bogotanos que tienen que desplazarse para llegar a sus sitios de trabajo, estudio o de actividades extras. Este sitio causó gran escándalo ya que, en su mayoría, las mujeres bogotanas que han sido ajenas a las violencias y de la guerra en nuestro país se dieron cuentan que esta clase de crímenes pueden suceder cerca a sus casas, zonas de estudio o de trabajo donde diariamente se transita.

El lugar del ataque a Rosa Elvira fue el Parque Nacional Enrique Olaya Herrera, inaugurado en 1934 por el presidente Enrique Olaya Herrera, es uno de los más importantes de la ciudad, por ser uno de los más antiguos. Cuenta con un terreno de 65 hectáreas que van desde la avenida Circunvalar a la carrera Séptima entre las calles 39 y 36 sur. El parque es de fácil acceso por cualquiera de los barrios aledaños, además de su ubicación, es un sitio atractivo para los estudiantes de las universidades cercanas como la Javeriana, Distrital, La Piloto, entre otros centros de educación, por ser una zona recreativa.

En la entrevista con Diana López, coordinadora de Fondo Lunaria, menciona porqué fue tan fuerte el impacto del hecho, más que todo en Bogotá.

El hecho de un acto tan violento y con tal sevicia, de tal magnitud, despreocupación y culpa del Estado tan cerca de nosotras, muy cerca de donde circula y se ubican un montón de mujeres diariamente, es ahí en el Parque Nacional al lado de las universidades y de muchos sitios más, es así, como las colombianas se vieron identificadas a poder sufrir cualquier tipo de ataques ya que fue aquí, en el corazón de Bogotá, en un lugar donde transita todo el mundo y yo creo que lo que muchos pensamos fue que "pude haber sido yo". (D. López, comunicación personal, 21 de febrero de 2019). 


\section{Mujeres accionando por Rosa Elvira Cely}

\subsection{Adriana Arandia Cely: de hermana a activista por los derechos de las mujeres en} Colombia

Adriana es una de muchas colombianas que han tenido que sufrir la pérdida de una de sus integrantes del grupo familiar por lo que anteriormente se conocía como crímenes pasionales, siendo víctimas de la violencia contra las mujeres y reflejando la indolencia del Estado por no garantizar una vida digna y libre de todo tipo de violencias contra la mujer.

Adriana es quien da inicio al segundo llamado para reactivar (lo que Rosa Elvira desde la primera llamada a la línea de emergencia había iniciado) la ruta para acceder al adecuado proceso de atención ante tal hecho registrado y para garantizar que este caso no quedara en la negligencia. Durante este proceso, Adriana pasa de ser una mujer con una vida totalmente distinta a la que tomaría en el momento de conocer lo acontecido por su hermana, es en ese momento que interviene en el asunto de la denuncia y de hacer un proceso de investigación al lado de su prima, ya que ningún ente ponía atención a lo sucedido; es en ese momento que juntas empiezan un trabajo complejo sin tener conocimientos previos sobre el tema judicial, empezaron a vigilar y a hacer seguimiento a los criminales.

Al cambiar su estilo de vida, Adriana pasó de ser estudiante de diseño de modas a luchar porque todo el dolor que vivió su familia no lo vivieran más familias, por las víctimas que pasaron silenciadas e ignoradas ante la justicia y por evitar posibles feminicidios a futuro, es ahí donde cambia su rumbo personal y académico y comienza a estudiar trabajo social y a trabajar en temas contra la violencia hacia la mujer (Caracol Radio, 2017).

Al vivir una situación con tal grado de sevicia y desprecio hacia un ser querido no solo por parte del agresor, sino de los entes públicos que no garantizaron la atención necesaria para salvar la vida de Rosa Elvira, además, de instituciones y comentarios que culpabilizaban a la víctima por pensamientos machistas, se motivó a luchar por el proceso de su hermana y por alzar la voz por las demás víctimas registradas por este mismo tipo de hechos.

Respecto a lo sucedido, Adriana en la entrevista contó cómo se convirtió en una activista:

Viendo las necesidades de nosotras inicialmente, que son las mismas necesidades que tienen todas las mujeres todos los días, las mujeres que son víctimas de cualquier tipo de violencia y es así como me convierto en defensora de los derechos de las mujeres en nuestro país, por lo que sucedió con nosotras, por lo que vemos que ha sucedido con tantas mujeres y que sigue sucediendo en nuestro país. (A. Arandia, comunicación personal, 23 de febrero de 2019).

Siendo una mujer activa en las problemáticas de feminicidios y líder dentro del movimiento de mujeres en Colombia, Adriana mencionó que la situación que vivió:

Es un enfrentamiento diario a una realidad, de lo que pasa en las mujeres y lo que le pasó a mi hermana, de una sociedad indolente, de una sociedad con memoria corta, momentánea en donde su indignamiento muchas veces queda ahí. (Caracol Radio, 2017). 
Situación que la impulsó a continuar la lucha contra un Estado y una sociedad para tomar acción; este fue el camino que vivió para su transformación a activista por los derechos de las mujeres y por una vida digna libre de violencias para las mujeres bogotanas y colombianas.

\subsection{Movimiento feminista bogotano accionando por Rosa Elvira Cely}

Al hablar de los grupos, colectivos, fondos, organizaciones y sociedad en general que participaron frente a este crimen, es necesario aclarar que solo se tomarán los que participaron en la ciudad de Bogotá por dos razones principales, la primera es que es la ciudad en donde ocurrieron los hechos y, la segunda, es que por ser la capital colombiana recae un peso y un activismo de la comunidad. En las primeras reacciones están los familiares, encabezados por Adriana Arandia Cely, luego las reacciones de diez estudiantes de la Universidad Nacional, quienes promovieron desde las redes sociales un plantón como medio para rechazar este tipo de actos por medio del hashtag \#NiunaMas; asimismo se unieron a la movilización "la Casa de la Mujer, Oxfam, Women's Link Worldwide, la periodista y subeditora judicial de El Tiempo Jineth Bedoya y otras organizaciones" (Sarralde, 2012, s.p.), entre otras más que en primera instancia se solidarizaron y se conmovieron ante tal crimen.

Es importante aclarar que el movimiento feminista que intervino frente a la muerte de Rosa Elvira no fue solo de Bogotá; así lo expreso Adriana Arandia Cely en la entrevista:

Yo creo que todos los movimientos que están en nuestro país participaron, no solamente en Bogotá porque esto fue a nivel nacional no solamente salieron a marchar el 3 de junio de 2012, sino que también se pronunciaron, sacaron su documento de sentido y tomaron acción para unirse y pedir la garantía de los derechos de las mujeres. (A. Arandia, comunicación personal, 23 de febrero de 2019).

De igual forma, en Bogotá fueron muchas las organizaciones que participaron, tales como:

La Casa de la Mujer, Cijusticia, Organización de Mujeres Feministas, cantidades no solamente de mujeres que están organizadas sino también hombres y mujeres de la sociedad que les tocó el caso de mi hermana, pero que a la vez tenían alguno de sus familiares o desaparecidos o habían muerto víctimas de cualquier violencia en nuestra ciudad. (A. Arandia, comunicación personal, 23 de febrero de 2019).

Esto reflejó el impacto que causó en la sociedad colombiana. De la misma forma, Diana López de Fondo Lunaria mencionó que existió un gran número de organizaciones que se pronunciaron, entre ellas "la Casa de la mujer, la batucada La Tremenda Revoltosa, la batucada Manada Callejera, la Corporación Colombiana de Teatro con la maestra Patricia Ariza, Ni Una Más, Mujeres por la Paz y la Red Nacional de Mujeres" (D. López, comunicación personal, 21 de febrero de 2019), quienes por medio del arte y de la música se hicieron presentes en las conmemoraciones, talleres, foros y demás espacios, como un medio de manifestación artística que exigía justicia por Rosa Elvira y las demás víctimas de feminicidios. 
De acuerdo a lo expuesto anteriormente en el marco teórico sobre los nuevos movimientos sociales (NMS) se puede establecer que el movimiento feminista bogotano que se activó por el caso de Rosa Elvira cumplió con el sentido de los nMs, ya que reconocen que buscaron el desarrollo y trasformación de la sociedad con nuevos parámetros, distintos a los antiguos movimientos, donde resaltaron los derechos humanos de las mujeres y buscaron la garantía de poder obtener la seguridad, justicia, equidad de género y el cumplimiento de una vida digna y libre de todo tipo de discriminaciones y violencias para las mujeres.

Con los nuevos movimientos se generó un cambio de ideología basado en la toma de conciencia que busca la trasformación de la situación de vulneración de la mujer. Es decir, se busca construir una nueva identidad que permita un mejor actuar. En Colombia, estos nuevos movimientos, en especial el feminista, buscan ser escuchados e interactuar con el Estado ante las vulneraciones, para así eliminar todas las barreras de silencio. Se buscó, también, incentivar una mayor participación y la construcción de una sociedad justa en donde las mujeres puedan gozar de una buena calidad de vida, tal como lo hicieron y aun lo hace el movimiento feminista colombiano al exigir justicia por Rosa Elvira, al alzar la voz, al querer una trasformación y al luchar por una sociedad equitativa y con justicia para que los crímenes que atentan contra el bienestar de las colombianas no queden en la impunidad.

Al hablar de las características de los Nuevos Movimientos Sociales, Mardones alude a que:

Los nuevos movimientos sociales pueden contribuir a solucionar los cuatro grandes problemas de la sociedad actual, a saber: 1) impulsar una economía de la moderación y el reparto igualitario, 2) promover unas nuevas relaciones con la naturaleza, basadas en el respeto y la conservación, 3) buscar la negociación como medio de solución de los conflictos nacionales e internacionales, y 4) cultivar la confianza en todo tipo de relación humana. (Mardones, 1996, p. 38).

En lo que se refiere al movimiento social feminista de Bogotá, frente al caso de Rosa Elvira, cumplió con lo descrito por Mardones ya que sí impulsó que todas las mujeres luchen por obtener una vida digna económica, social y culturalmente libre de todo tipo de violencias, es decir, una vida sin discriminaciones.

En cuanto al promover las relaciones con la naturaleza, el movimiento se vinculó con el medio ambiente dentro de sus acciones colectivas en zonas como el Parque Nacional de Bogotá, con ofrendas y promoviendo actividades de cultivar árboles y rosas. Asimismo, mantuvo negociaciones respetuosas y de solidaridad, se tiene en cuenta la unión que este generó por exigir el cumplimiento de los derechos humanos, a la vez resaltar la sororidad brindada por varias mujeres hacia el movimiento. Finalmente, la confianza brindada entre las mujeres dentro del movimiento, la ética en la cual se destacó la manera en que el movimiento y sus acciones se expanden buscando solucionar la problemática del feminicidio y de la ausencia de justicia por parte del Estado a estos casos que, en su momento, eran denominados crímenes pasionales.

Al mencionar los integrantes del movimiento feminista bogotano y sus reacciones frente a lo sucedido a Cely, aparecen los siguientes actores: la Ruta Pacífica de las Mujeres, el cual es 
un movimiento feminista y pacifista colombiano en pro de los derechos de las mujeres y la construcción de entornos libres de violencias e injusticias. Desde que se supo lo acontecido por Rosa Elvira fue de las primeras en generar públicamente un comunicado en donde expresaron su dolor y rabia frente a lo ocurrido el 23 de mayo del 2012 a Rosa Elvira. De la misma forma, se pronunciaron: Olga Amparo Sánchez, representante de la Casa de la Mujer; Mónica Roa, directora de la ong Women's Link; el defensor del pueblo, Volmar Pérez; Diana Montealegre, representante de la ONG británica Oxfam; Diego Molano, director del Instituto Colombiano de Bienestar Familiar (ICBF); Iván Cepeda Castro, defensor de los derechos humanos; Jorge Luis Palacios de la Corporación de Investigación y Acción Social y Económica (CLASE), entre muchos más personajes involucrados frente a este caso, ya bien estén dentro o fuera del movimiento feminista bogotano.

Estos comunicados frente a la violación y muerte de Rosa Elvira reflejaron cómo diversas instituciones coincidieron en que esta clase de actos no deben presentarse más en Colombia. En este punto se destacó la solidaridad de cada persona, entidad, institución de la sociedad en general que ayudaron a visibilizar este hecho y la violencia contra las mujeres, la cual era un hecho que cualquier mujer estaba y aún está expuesta en Colombia. Estos comunicados ayudaron a la difusión de este acto que anteriormente era silenciado.

En este punto, los comunicados y los medios de comunicación jugaron un papel muy importante, ya que dieron ejemplo a la sociedad, Arandia menciona que el caso mediático "realmente ayudó un poco a que la justicia le pusiera el ojo a las violencias contra las mujeres y específicamente con el caso de Rosa Elvira Cely", asimismo menciona que generó una parte negativa ya que "dejan de un lado la humanidad y la importancia de la familia y del ser humano y venden esa noticia de una manera denigrante y morbosa" (A. Arandia, comunicación personal, 23 de febrero de 2019). Es decir, los medios de comunicación en cierto punto abusaron de la confianza y del luto de la familia Cely al ingresar al hospital y tomar fotos que eran impactantes, pero, a su vez, ayudó a mostrar una cruda realidad, una situación que motivó a colectivos y al movimiento feminista bogotano a salir a las calles indignados a rechazar este tipo de violencia contra las mujeres, a exigir justicia por todas las víctimas y sus casos impunes y a unirse por un país en formación a una sociedad libre de violencia de género (A. Arandia, comunicación personal, 23 de febrero de 2019).

\section{La conmemoración, más allá de una acción}

Una de las acciones colectivas que más se resalta en la investigación es la conmemoración de cada año en el mes de mayo por la muerte de Rosa Elvira en el Parque Nacional de Bogotá, como un medio que reconoce la problemática que viven a diario las mujeres y que por medio de su historia buscan animar a la sociedad a cambiar de pensamiento y a luchar por una sociedad libre de violencias contra las mujeres. Estas conmemoraciones afirmaban la unión de varios grupos, colectivos, instituciones y civiles que accionaban en el movimiento feminista bogotano 
y que estaban unidos por una misma indignación, con un mismo fin, para fortalecer e incentivar una trasformación en la sociedad colombiana, frente a situaciones en las cuales las mujeres son las principales víctimas.

De acuerdo con Ibarra, Martí y Gomá (2002) y su concepto de la capacidad de las acciones colectivas, se evidenció que las acciones colectivas presentadas en Colombia por el movimiento feminista que reaccionó por la muerte de Rosa Elvira sí cumplieron esta definición, ya que lograron trasmitir el mensaje a la sociedad y que varios sujetos se unieran a esta causa por solidaridad y porque identifican que es una problemática que puede el día de mañana corresponder a cualquier mujer de su entorno.

Es decir, fue un hecho que no es ajeno, que diariamente sucede y que se busca hacer frente y lograr, mediante acciones, que se siga repitiendo y quedando impune y, de esta manera, castigar toda clase de violencia contra las mujeres en Colombia. Asimismo, Funes y Monferrer (2003) concuerdan al decir que las acciones colectivas presentadas en Colombia durante el 2012-2015 por la muerte de Rosa Elvira, presentaron a una población motivada por un mismo fin en general, el cual es que las mujeres puedan tener una vida digna y libre de cualquier tipo de discriminación y violencia hacia ellas, mediante diversas acciones como conmemoraciones que ejercieron un protocolo de organización y movilización por las partes participantes para el tan anhelado cambio social.

Según la tipología impuesta por Tarrow (1997), se puede establecer que el movimiento feminista bogotano se presenta con acciones colectivas por Rosa Elvira Cely como la manifestación pública organizada, ya que desde el momento de conocer el brutal crimen reaccionaron de manera pacífica y organizada, comunicando a la sociedad y realizando una convocatoria para la participación en el Parque Nacional, tal como en primera instancia lo realizó la Ruta Pacífica de las Mujeres en el Comunicado de prensa $n .^{\circ}$ 7-2012, en donde expresaron:

Nos unimos a la jornada de duelo y dignidad a realizarse en Bogotá este domingo 3 de junio a las 10 a.m. en el Parque Nacional. Expresamos nuestro rechazo a estos actos de violencia contra las mujeres. Exigimos investigación y juzgamiento de los responsables de este horrendo crimen. Exigimos calles y ciudades seguras, libres y sin violencias hacia las mujeres y niñas. (Ruta Pacífica de las Mujeres, 2012).

De la misma manera, lo realizaron diez estudiantes de la Universidad Nacional, quienes promovieron desde las redes sociales la marcha para el día 3 de junio con la etiqueta \#NiUnaMás, rechazando todo tipo de violencia hacia las mujeres (Sarralde, 2012).

Al hablar de la primera conmemoración, esta fue realizada días después que los medios de comunicación informaran lo sucedido, es allí donde un grupo de estudiantes y egresados de la Universidad Nacional en Bogotá decidieron crear una página en Facebook con el nombre de Ni una Más, de esta manera convocaron a la sociedad en general para que se unieran y asistieran al Parque Nacional como rechazo a lo sucedido (Racines, 2012). Este hecho fue acogido por gran número de personas que decidieron asistir por los derechos de las mujeres, allí se reunieron 
movimientos feministas, organizaciones y colectivos de mujeres, instituciones, figuras públicas y ciudadanos del común que expresaban su solidaridad y embocaban justicia.

Es importante recordar que frente a esta situación las redes sociales y las herramientas web fueron importantes en la difusión y convocatoria a marchar ya que, por medio de correos electrónicos, mensajes en redes sociales, cadenas de imágenes y llamadas telefónicas, se logró incentivar a la sociedad a que este suceso no pasara en alto y generar una manifestación pública organizada sin violencia ni acciones directas disruptivas. Entre las plataformas que más se usaron para promover acciones colectivas fueron Facebook y Twitter, para reafirmar que Colombia no tolera más este tipo de actos (Radio Santa Fe, 2012).

Al pasar los años, esta acción se convirtió en conmemoraciones, las cuales fueron un medio de acción, ya que la sociedad se organizó con camisetas con mensajes alusivos en contra de este tipo de hechos, afiches, carteleras, pancartas con mensajes de ¡Ni Una Más!, exigiendo justicia ante todos los casos impunes de feminicidios.

Es importante mencionar, que en las conmemoraciones las expresiones artísticas fueron el pilar para el reconocimiento y la expresión del dolor en diferentes manifestaciones con poemas, canciones en especial de rap como lo realizaron la Batucada Tremenda Revoltosa y la Batucada Manada Callejera y por medio de la actuación estuvo presente la Corporación Colombiana de Teatro. Entre otras acciones presentes en la conmemoración como la ofrenda floral, performance, presentaciones culturales, conversatorios de sobrevivientes a feminicidios, testimonios entre otros hechos que en la actualidad se mantienen vigentes. (El Espectador, 2015).

Diana López de Fondo Lunaria mencionó que las acciones colectivas por Rosa Elvira se basaron en la:

Conmemoración del día de los hechos y la resignificación del espacio. Hay unas organizaciones que sabemos siempre qué se hace, también, recordar que la que manda la parada es Adriana, ella es quien dice las fechas, hay veces que sabemos que la fecha no nos va a coincidir muy bien, se intenta hacer un domingo para que puedan subir varias mujeres, como que se pueda hacer algo bien bonito e importante, pero no siempre participan las mismas, a veces participan más, a veces menos, eso depende de quienes estén convocando, de cómo se convoquen, de cómo se haga la convocatoria y qué se piensa hacer allí en el lugar. (D. López, comunicación personal, 21 de febrero de 2019).

Es decir, que la principal acción colectiva presentada durante el 2012-2015 fue la conmemoración de la muerte de Rosa Elvira, en la que se plasmó la voluntad de movimientos, organizaciones, colectivos y demás a no olvidar este y ningún otro feminicidio en Colombia, a que ninguno quede impune y que se logre garantizar la vida y seguridad de las mujeres.

\section{Logros y beneficios del accionar feminista por Rosa Elvira en Bogotá y en el resto de Colombia}

Uno de los logros más sobresalientes en el accionar del movimiento feminista en Bogotá fue el del proyecto de ley para castigar este tipo de hechos como feminicidios en Colombia. 
Es importante aclarar que, aunque ya estaba la Ley 1257 de 2008, "por la cual se dictan normas de sensibilización, prevención y sanción de formas de violencia y discriminación contra las mujeres", esa tipificación no fue suficiente, ya que se seguían presentando actos de extrema agresión hacia las mujeres. Es por esto que el movimiento que se activó por Rosa Elvira luchó por una ley que complementara la Ley 1257 de 2008 y que castigara explícitamente este delito como un feminicidio, como lo fue la Ley 1761 de 2015 (Naranjo, 2015).

Arandia, al ser uno de los personajes más importantes dentro de la movilización, mencionó que además de la ley se encaminaron más acciones sobre el tema de prevención y en la atención para la garantía plena de los derechos, que no solo cubren el periodo a investigar del 2012 a 2015, sino que aun en la actualidad es un tema que se sigue trabajando. Asimismo, indicó que este proyecto de ley que hoy en día es la Ley Rosa Elvira Cely es un legado para futuras generaciones, así lo expresó:

Sería lo único que podemos dejarles a las nuevas generaciones, a nuestras hijas, hijos, a hombres y a mujeres. Es el poder trabajar no solamente por haber creado la ley, sino por crear una herramienta que podemos utilizar en procesos de prevención, educativos, pedagógicos y demás. (A. Arandia, comunicación personal, 23 de febrero de 2019).

De esta manera, se recalcó que la acción colectiva más importante en toda la movilización sin duda fue la Ley Rosa Elvira Cely, producto de la lucha desde el 2012 hasta el 2015, ya que durante este periodo "fue un tiempo de presión de muchos colectivos y organizaciones de mujeres, encabezadas por Adriana Arandia Cely y con asesorías de abogadas y demás, además de contar con la facilitación de algunas mujeres en el congreso" (D. López, comunicación personal, 21 de febrero de 2019). Asimismo, señaló la importancia de la ley no solo en ámbitos legales y de política pública, sino en ámbitos emocionales y el significado que esta brinda a la sociedad en específico a las mujeres en Bogotá y en el resto de Colombia.

Es menester mencionar que después de lo sucedido con Rosa Elvira en Bogotá se creó la Secretaría Distrital de la Mujer el día 28 de junio de 2012 en busca de garantizar los derechos de las mujeres en Bogotá y de eliminar discriminaciones y todo tipo de violencias (Secretaría Distrital de la Mujer, 2013). Arandia comenzó a trabajar en la Secretaría de la Mujer en su lucha porque las mujeres tengan justicia y la asesoría necesaria en la cual las entidades correspondientes hacen prevención y seguimiento. Al hablar de la primera administración en donde se ejecutó la Secretaría se menciona que:

Tuvo énfasis en las organizaciones de mujeres y se tiene en cuenta, desde ese momento, las mujeres indígenas, las mujeres afros, la diversidad que existe para trabajar en el tema de empoderamiento, de emprendimiento y demás, pues no son suficientes, pues tristemente cuando hay cambio de administración, cuando hay esta ruptura yo creo que se pierde un poco el ideal, se pierde un poco quizás la línea que debería seguirse y es volver a trabajar los derechos de las mujeres. (A. Arandia, comunicación personal, 23 de febrero de 2019). 
Aún hoy en día, a pesar de que exista esta secretaría, se debe hacer presión para garantizar el buen trabajo en pro de las mujeres con un personal psicojurídico, que garanticen que las mujeres víctimas cuenten con la ruta de atención necesaria.

Además de la ley, otro logro que se puede destacar fue la creación de la Cátedra de Género que fue una de las solicitudes y una de las obligaciones que salen de la misma ley, además, de ser una de las solicitudes que la familia Cely tenía, ya que se preguntaban cómo los colegios, las universidades y centros de educación no contaban con una cátedra de género para poder orientar a los jóvenes frente a temáticas sobre la violencia contra las mujeres, para formar a la comunidad y lograr prevenir y sancionar diversas situaciones a las que las mujeres son expuestas diariamente (dhColombia, 2018).

Desde el momento de lo acontecido por Rosa Elvira, Adriana y su familia lucharon por una cátedra de género que eduque y complemente la Ley Rosa Elvira Cely. De esta forma, la Ley 1761 "obliga a que el Ministerio de Educación implemente esa cátedra de género en colegios y universidades y, obviamente, al aparato judicial" (Buitrago, 2017). En este punto, se destacó el trabajo de Arandia por educar y por impartir un discurso que evite la violencia contra la mujer; es por esto que decidió aceptar la propuesta de plasmar la realidad que vivió su hermana y su familia durante el duro proceso que conllevó la muerte de Rosa Elvira. Es allí donde Arandia decide formar equipo con Fernando González y realizaron el libro La vida es Rosa: el oscuro amanecer de Rosa Elvira Cely en el Parque Nacional. Para Arandia:

El libro es una acción colectiva, porque no fue hecha por un solo autor, por un solo escritor, no fue escrita por una sola persona, González fue nuestro guía, porque la historia se la contamos nosotros, la historia es nuestra, el dolor es nuestro y está reflejado a muchas mujeres, este libro es un trabajo pedagógico en muchos espacios, es decir, es una acción colectiva hecha colectivamente. (A. Arandia, comunicación personal, 23 de febrero de 2019).

Este es un instrumento que dio pautas para ser consciente que son muchas las mujeres que en su momento han sido víctimas de violencia.

A este proceso del libro se unieron el Fondo Lunaria, ya que a partir de la publicación del libro se hizo presente en talleres y en realizar charlas con Adriana Arandia, Fernando González y con su equipo investigativo, "lo que hicimos fue vincularnos en la difusión del libro y en una relación mucho más cercana con lo que hoy se llama Ni Una Más” (D. López, comunicación personal, 21 de febrero de 2019). De esta manera, López mencionó que se empezó un acompañamiento a Arandia con un vínculo de solidaridad. Es importante mencionar que:

La función de los talleres es contar un poco lo que se hizo sobre el libro, ya que el libro es un relato de mujeres que han sido violentadas a lo largo de su vida, donde las mujeres jóvenes lo toman como una herramienta para desarrollar memoria, para contar lo que nos pasa a las mujeres y para buscar justicia. (D. López, comunicación personal, 21 de febrero de 2019). 
Es básicamente lo que realizó Arandia y González en los talleres, las lectoras desde la primera página se darán cuenta de la realidad y la posición del Estado frente a los casos de violencia hacia las mujeres.

\section{1. ¡Ni Una Más! Generando conciencia en pro de una vida digna y libre de violencia contra las mujeres}

Ni una Más o Ni una Rosa Más es una campaña que nació por la indignación del atentado a Rosa Elvira Cely, por su muerte y por la falta de atención del Estado ante una víctima que sufrió una tortura que marcó al país con un antes y un después. Es un ejemplo de lo acontecido por Cely, de lo que pueden sufrir las colombianas y el temor que al denunciar no se brinden la atención necesaria. Es por esto que unos estudiantes y egresados de la Universidad Nacional deciden utilizar las redes sociales, en específico Facebook, para convocar y movilizar a la sociedad bajo la consigna de Ni una Más, para ejercer presión al Estado y exigir que se cambie la estructura de un sistema que ataca e invisibiliza los daños a las mujeres.

Adriana, líder y promotora de Ni una Más, mencionó que surge con el fin de poder orientar a las mujeres en términos de acceso a la justicia y las rutas de atención a seguir, además, de brindar el apoyo a los familiares de la víctima para trabajar en temas de reparación por medio de conmemoraciones, de visibilizar la historia de las mujeres víctimas de violencia de género. Así mismo lo indicó Adriana:

El reconocer su historia, el reconocer que sobrevivieron y están luchando y que pueden mostrar esa historia a otras mujeres que se pueden sanar a sí mismas pero que también pueden ayudar a las otras, pues porque todas no tenemos las mismas capacidades y quizás ni siquiera las mismas oportunidades, entonces, lograr crear unos productos en pro de ese trabajo con las mujeres, pero además organizar a estas mujeres para que ellas sean las que repliquen y sean las que crean su propia red de apoyo, creo que es lo fundamental en este caso. (A. Arandia, comunicación personal, 23 de febrero de 2019).

Es necesario mencionar que esta campaña de "Ni una Más" realizó una carta al Estado, a la sociedad colombiana y al mundo frente a la situación de muertes a mujeres y la violencia que diariamente son expuestas, la carta dice:

Exigimos al Estado que nos garantice la posibilidad de habitar tranquilas nuestras casas, las calles, las escuelas, el trabajo, la ciudad a cualquier hora. Exigimos que nos explique las inconsistencias que encubren la desidia con la que fue atendida Rosa, que explique la ineficacia de la fuerza pública por no atender de forma oportuna al llamado, y nos explique las decisiones de las instituciones de salud que no tomaron en consideración la gravedad del caso. Lo exigimos no solamente por el caso de Rosa, sino porque estamos seguras y seguros que este es el viacrucis de miles de mujeres que tienen que afrontarse con este tipo de comportamientos, e incluso, con la estigmatización que las convierte injustamente en culpables cuando en realidad, son las víctimas. Las mujeres tenemos derecho a un vivir libre de violencias. (Ni Una Más, 2012). 


\section{Conclusiones}

Es transcendental mencionar que el movimiento feminista bogotano, durante el 2012-2015, trabajó en búsqueda de erradicar todo tipo de violencia hacia las mujeres, exigiendo justicia y protección por parte del Estado para las mujeres víctimas. En este punto, el movimiento se complementó con las acciones colectivas que dan paso a la ejecución, estrategias y desarrollo de alianzas para lograr sus objetivos y obtener unos resultados para el beneficio de las mujeres no solo en Bogotá, sino en toda Colombia.

El caso de Rosa Elvira fue el ejemplo de la violencia a las que las mujeres son expuestas diariamente, en un país en donde el Estado no ofrecía las garantías ni la protección a las mujeres víctimas de distintos tipos de violencia, en especial de las muertes a mujeres por condición de género, como se reflejó en el caso de Rosa Elvira, donde se demuestra la ausencia estatal que produjo y la negligencia en ámbitos de atención, salud y justicia.

Para la presente investigación los integrantes del movimiento feminista bogotano que participaron y realizaron acciones ante el caso de Rosa Elvira Cely fueron: Adriana Cely, Fondo Lunaria, Ni una Más y el Movimiento Internacional Feminismo Artesanal, los cuales participaron en la entrevista semiestructurada para el presente trabajo de investigación, cada uno destacó el papel de la unión de personas, colectivos, fondos y demás grupos de mujeres que se unieron y brindaron un accionar para exigir justicia y evitar que este crimen quedara en la impunidad.

Frente a estas consideraciones, en el caso de Rosa Elvira se evidenció que el movimiento feminista bogotano reaccionó desde el primer momento cuando los medios de comunicación dieron la cobertura de lo sucedido, es allí cuando se generó una conmoción nacional en la que se involucraron varios autores, tanto del ámbito público como privado. Es en este punto que se reconoció la principal acción colectiva que fue la de la movilización social, donde grupos, colectivos, fondos, organizaciones y demás personas en general se reunieron en el Parque Nacional, al difundirse la noticia a finales de mayo para la realización del plantón del 3 de junio de 2012 . Es ahí donde la sociedad, por medio de las redes sociales y comunicados, logró iniciar la primera acción convocando e invitando a la comunidad a participar por Rosa Elvira.

Esta acción de generar, en primera instancia, un plantón reflejó cómo el movimiento feminista bogotano reaccionó de manera inmediata frente a este crimen, así se buscó comunicar y trasmitir que esta realidad debe ser evaluada por el Estado con el objetivo de que lo sucedido no quede en el olvido, ni se silencie una problemática que atenta diariamente a las mujeres en Bogotá y en Colombia. Frente a esta acción se logró percibir cómo la solidaridad es uno de los principales valores ejercidos, se lograron vincular personas de diversos ámbitos e ideales de la sociedad en un solo parque con un mismo fin, generar justicia y evitar que sigan atentando contra la vida de las mujeres.

Es significativo aclarar que la acción más importante es la que realizaron en el primer plantón, puesto que esta acción se convertiría en una conmemoración anual y un espacio representativo, primero porque fue donde ocurrió el crimen; segundo, por ser un lugar central y 
transitado en Bogotá, además de tener el espacio necesario para toda la población asistente y así recrear escenarios que hicieron honor a Rosa Elvira. Es ahí donde por medio de flores, expresiones religiosas, artísticas, musicales, teatrales y demás, manifestaron su dolor, el recuerdo por todas las víctimas de feminicidios y se exigió la presencia del Estado y el adecuado seguimiento frente a los feminicidios en el país.

Es de admirar el movimiento feminista bogotano y los demás participantes que se unieron a apoyar estas acciones, ya que Bogotá asumió el protagonismo y tomó conciencia colectiva ante las vulneraciones, discriminaciones, violencias y muertes a mujeres, logrando generar una organización y movilización que marcó históricamente el futuro de Bogotá y del resto de Colombia. Es allí donde todo el movimiento compartió una misma meta, sintieron propio el problema, establecieron formas de actuar y de accionar, todo esto guiado a generar justicia, a que esta clase de crímenes no sigan ocurriendo y lograr la trasformación del entorno donde las mujeres vivan libres y seguras. Referente a la integración del movimiento feminista bogotano, este contó con el apoyo del sector público y privado, es decir, se contó con una participación múltiple que no solo cubrió el sector de Bogotá, sino que trascendió al resto del país.

Por consiguiente, al hablar de los logros del accionar del movimiento feminista bogotano, respecto al caso de Rosa Elvira, podemos evidenciar que el mayor logro fue la Ley 1761 de 2015 o Ley Rosa Elvira Cely, ya que por medio de las acciones colectivas se logró captar la atención no solo de Colombia, sino también del Estado, abriéndose paso a ser uno de los temas más trascendentales del país y, de esta manera, consiguió captar la atención de los medios de comunicación y del Congreso.

Es importante mencionar que esta ley tipifica al feminicidio como un delito autónomo para sancionar y a la vez prevenir las violencias a las que están expuestas las colombianas, aunque antes ya se contaba con una ley, como lo era la Ley 1257 de 2008 para la sensibilización, prevención y sanción de diversas formas de violencia hacia la mujer, no era suficiente y fue por eso que la Ley Rosa Elvira entra a complementarla. Estas dos leyes no son suficientes, ya que hoy en día se siguen presentando altos niveles de violencia contra las mujeres con impunidad, aún falta más compromiso estatal por hacer cumplir y llevar a cabo estas leyes para garantizar una sociedad segura para las mujeres. Un ejemplo de que el Estado no es garante de los derechos de las mujeres es el mismo caso de Rosa Elvira con su agresor, quien ya tenía un registro criminal pero que aun así no cumplió condena y quedó libre. Es ahí donde se debe presionar al Estado para que haga bien su labor y que desde los primeros indicios de estos criminales se ejerza un castigo y seguimiento para así evitar que posibles feminicidas anden libres y poniendo en riesgo la vida de las colombianas.

Gracias a la suma de manifestaciones del movimiento feminista bogotano y presiones ejercidas en el 2012-2015 al Estado para exigir justicia y castigo a este feminicida, Velasco es condenado. La suma de los delitos da 90 años de cárcel, pero en Colombia la pena máxima es de 60 años en prisión, además Velasco acepta todos los cargos, y por eso la justicia colombiana le dio 
una rebaja y en total pagará 48 años de prisión. Al aceptar la condena expresó: "Quiero aprovechar para pedir perdón a la sociedad, a los familiares de la víctima. Siento mucho dolor. Le pido a Dios y a todos que me perdonen" (Semana, 2012b). Hecho que refleja que los feminicidas no le temen a la justicia y que no piensan que serán juzgados por una sociedad que anteriormente invisibilizaba, ignoraba y callaba este tipo de actos.

Asimismo, es importante mencionar la campaña Ni una Más como una de las campañas más significativas para rendir homenaje a Rosa Elvira y para pronunciarse en contra de muertes a mujeres en condiciones indignas y por motivo de género. Esta campaña es significativa ya que se involucró dentro de las acciones colectivas presentadas por el movimiento, además de haber sido uno de los hashtags más sobresalientes en las redes sociales al manifestarse sobre la temática de los feminicidios. Esta es una campaña que hasta el día de hoy sigue vigente y es de gran importancia, ya que se usa frente a las violencias que sufren las mujeres.

Uno de los resultados que más llama la atención fue la creación del libro La vida es Rosa: el oscuro amanecer de Rosa Elvira Cely en el Parque Nacional, ya que es un instrumento que sirve para reflexionar la situación de Rosa Elvira y su familia, al mismo tiempo de ser utilizada como una herramienta para brindar cátedras académicas. Es en este punto que se resalta la actividad de charlas y conversatorios de la vida es rosa, realizados por Adriana Arandia y Fernando González Santos como una de las herramientas pedagógicas que sobresalen de todo el dolor causado por el feminicidio de Rosa Elvira, para brindar a ayuda y orientación a todas las colombianas.

Finalmente, el movimiento feminista bogotano logró iniciar una trasformación histórica en Colombia, ya que asumió el protagonismo para decir basta ya de muertes a mujeres por su condición de serlo, es decir, es gracias al accionar que se logra tipificar este tipo de hechos como feminicidios dentro de la legislación colombiana, trabajando por evitar injusticias, porque sus acciones colectivas fueron guiadas a la conservación de los derechos y la justicia para Rosa Elvira y todas las demás víctimas de este flagelo.

\section{Referencias}

Ayala, L. (2019). Las acciones colectivas del movimiento feminista de Bogotá frente al caso de Rosa Elvira Cely 2012-2015 (trabajo de grado). Universidad de Ibagué, Ibagué, Colombia.

Buitrago, J. (18 de agosto de 2017). Habla hermana de Rosa Elvira Cely, víctima que conmocionó al país. El Tiempo. Recuperado de https://www.eltiempo.com/bogota/entrevista-de-adriana-cely-hermanarosa-elvira-cely-sobre-violencia-contra-la-mujer-121374

Caputi, J., \& Russell, D. (1992). Feminicidio: Sexismo terrorista contra las mujeres. En Jill Radford \& Diana E. H. Russell (Eds.). Femicide: The Politics of Woman Killing [Feminicidio. La política del asesinato de las mujeres]. Nueva York, Estados Unidos: Twayne Publishers.

Caracol Radio. (12 de abril de 2017). Adriana Arandia Cely, cambió su vida tras el feminicidio de su hermana Rosa Elvira Cely. Caracol Radio. Recuperado de https://alacarta.caracol.com.co/ audio/097RD130000000503854/ 
Colombia. Ley 1257 de 2008. Por la cual se dictan normas de sensibilización, prevención y sanción de formas de violencia y discriminación contra las mujeres.

Colombia. Ley 1761 de 2015. Por la cual se crea el tipo penal de feminicidio como delito autónomo y se dictan otras disposiciones.

dhColombia. (24 de mayo de 2018). 6 años del feminicidio de Rosa Elvira Cely. dhColombia. Recuperado de http://www.dhcolombia.com/2018/05/24/6-anos-del-feminicidio-de-rosa-elvira-cely/

El Espectador. (28 de mayo de 2015). Bogotá marchará contra el feminicidio bajo la campaña “iNi una Rosa más!”. El Espectador. Recuperado de https://www.elespectador.com/bogota/bogota-marcharacontra-el-feminicidio-bajo-la-campana-ni-una-rosa-mas-article-563269/

El Tiempo. (15 de mayo de 2016). Los otros crímenes de Javier Velasco, asesino de Rosa Elvira. El Tiempo. Recuperado de https://www.eltiempo.com/archivo/documento/CMS-16593909

Funes Rivas, M.J, \& Monferrer Tomás, J. (2003). Perspectivas teóricas y aproximaciones metodológicas al Estudio de la participación. En Funes Rivas, M.J. y Adell Argiles, R. (Eds.), Movimientos Sociales: cambio social y participación (pp. 21-58). Madrid, España: UNED.

González Santos, F. (2014). La vida es Rosa: el oscuro amanecer de Rosa Elvira Cely en el Parque Nacional, 2014. Bogotá, Colombia: Controversia Editorial.

Huertas, O., \& Jiménez, N. (2016). Feminicidio en Colombia: reconocimiento de fenómeno. Pensamiento Americano, 9(16), 110-120. https://doi.org/10.21803/pensam.v9i16.71

Ibarra, P., Martí, S., \& Gomá, R. (2002). Creadores de la democracia radical. Movimientos sociales y redes de políticas públicas. Barcelona, España: Icaria editorial.

Iribarne, M. (2015). Feminicidio (en México). Eunomía, (9), 205-223. Recuperado de https://e-revistas. uc3m.es/index.php/EUNOM/article/view/2822

Lagarde, M. (2009). El feminicidio, delito contra la humanidad. En Feminicidio, justicia y derecho. México: Comisión Especial para Conocer y Dar Seguimiento a las Investigaciones Relacionadas con los Feminicidios en la República Mexicana.

Mardones, J.M. (1996). Los nuevos movimientos sociales y la sociedad moderna. En Mardones, J.M (director), 10 palabras clave sobre movimientos sociales. Navarra, España: Verbo Divino.

Monárrez Fragoso, J. E. (2002). Feminicidio sexual serial en Ciudad Juárez: 1993-2001. Debate Feminista, 25, 279-305. Recuperado de http://repositorio.ciem.ucr.ac.cr/handle/123456789/59

Naranjo, A. (07 de junio de 2015). Ley Rosa Elvira Cely: ¿estrategia integral frente a la violencia contra las mujeres? Razón Pública. Recuperado de https://razonpublica.com/ley-rosa-elvira-cely-estrategiaintegral-frente-a-la-violencia-contra-las-mujeres/

Kirino, N. (2003). Gurotesuku. Tokyo: Bungei Shunju.

Neiman, G., \& Quaranta, G. (2006). Los estudios de caso en la investigación sociológica. En Vasilachis de Gialdino (coord.), Estrategias de investigación cualitativa (pp. 213-237). Buenos Aires, Argentina: Gedisa.

Ni una Más. (2012). Por el derecho a la vida ¡Ni una ROSA más! Carta al Estado y a la sociedad colombiana y al mundo. Bogotá. 
Notiagen. (04 de junio de 2012). Masiva manifestación en Bogotá: Ni una Rosa más. Notiagen. Recuperado de https://notiagen.wordpress.com/2012/06/04/masiva-manifestacion-en-bogota-ni-una-rosamas/

Racines Arévalo, K. (2 de junio de 2012). Repudio por el crimen de Rosa Elvira Cely: ¡Ni una Rosa más! Semana. Recuperado de https://www.semana.com/nacion/articulo/repudio-crimen-rosa-elviracely-ni-rosa-mas/258953-3/

Radio Santa Fe. (31 de mayo de 2012). Redes sociales repudian asesinato de Rosa Elvira Cely en el Parque Nacional. Radio Santa Fe 1070 Bogotá. Recuperado de https://www.radiosantafe.com/2012/05/31/ redes-sociales-repudian-asesinato-de-rosa-elvira-cely-en-el-parque-nacional/

Ruta Pacífica de las Mujeres. (2012). Rabia e indignación por el asesinato de Rosa Elvira (Comunicado de prensa n. $^{\circ}$ 7). Recuperado de https://rutapacifica.org.co/wp/rabia-e-indignacion-por-el-asesinatode-rosa-elvira/

Sandoval Acosta, G. (2012). Acciones colectivas del movimiento de mujeres y del movimiento feminista en Cali: apuntes desde la historiografía feministas. Revista CS, (10), 55-90. https://doi.org/10.18046/ recs.i10.1355

Sarralde, M. (30 de mayo de 2012). Promueven plantón para rechazar la brutal violación a Rosa Elvira Cely. El Tiempo. Recuperado de https://www.eltiempo.com/archivo/documento/CMS-11913592

Secretaría Distrital de la Mujer. (26 de junio de 2013). Secretaría Distrital de la Mujer Celebra un año de su creación. Recuperado de http://portalantiguo.sdmujer.gov.co/inicio/57-noticia-de-primera-pagina1

Semana. (1 de junio de 2012a). La muerte de Rosa Elvira Cely, un crimen abominable. Semana. Recuperado de https://www.semana.com/nacion/articulo/la-muerte-rosa-elvira-cely-crimen-abominable/258867-3/

Semana. (26 de diciembre de 2012b). Javier Velasco: 48 años de cárcel por caso Rosa Elvira Cely. Semana. Recuperado de https://www.semana.com/nacion/articulo/javier-velasco-48-anos-carcel-caso-rosaelvira-cely/326821/

Tarrow, S. (1997). El poder en movimiento. Movimientos Sociales, Acción colectiva y política. España: Alianza.

Varela, N. (2008). Feminismo para principiantes. Barcelona, España: editorial B de Bolsillo.

Villareal Larrinaga, O., \& Landeta Rodríguez, J. (2010). El estudio de casos como metodología de investigación científica en dirección y economía de la empresa. Una aplicación a la internacionalización. Investigaciones Europeas de Dirección y Economía de la Empresa, 16 (3), 31-52.

Yin, R. (1989). Case Study Research: design and Methods, Applied social research. Newbury Park CA: Sage Publications. 